\title{
HOMOGENEOUS PHASE OF COEXISTENCE OF SPIN-TRIPLET SUPERCONDUCTIVITY AND FERROMAGNETISM
}

\author{
D. V. Shopova, D. I. Uzunov \\ CPCM Laboratory, Institute of Solid State Physics, \\ Bulgarian Academy of Sciences, BG-1784 Sofia, Bulgaria \\ (Received June 23, 2003; received in final form September 15, 2003)
}

\begin{abstract}
The coexistence of a homogeneous (Meissner-like) phase of spin-triplet superconductivity and ferromagnetism is investigated within the framework of a phenomenological model of spin-triplet ferromagnetic superconductors. The results are discussed in view of their application to such metallic ferromagnets as $\mathrm{UGe}_{2}, \mathrm{ZrZn}_{2}$, URhGe, and Fe.
\end{abstract}

Key words: superconductivity, ferromagnetism, phase diagram, order parameter profile.

PACS number(s): 74.20.De, 74.20.Rp

\section{INTRODUCTION}

Experiments at low temperatures and high pressure have indicated the coexistence of ferromagnetism and superconductivity in the metallic compounds $\mathrm{UGe}_{2}[1-3]$, $\mathrm{ZrZn}_{2}$ [4], URhGe [5] and also in Fe [6]. In contrast to other superconducting materials (see, e.g., Refs. $[7,8]$ ), in these metals the phase transition temperature to the ferromagnetic state is higher than the phase transition temperature to the superconducting state and the superconductivity not only coexists with the ferromagnetic order but is enhanced by it. It is widely accepted $[1,10]$ that this superconductivity can be most naturally understood as a spin-triplet rather than a spin-singlet pairing phenomenon (see, e.g., Ref. [9]). The experiments [6] on a high-pressure crystal modification of Fe, which has a hexagonal closed-packed structure, are also interpreted [1] in favour of the appearance of the same unconventional superconductivity. Note, that both vortex and Meissner superconductivity phases [6] are found in the high-pressure crystal modification of Fe where the strong ferromagnetism of the usual bcc iron crystal probably disappears [10].

The phenomenological theory that explains the coexistence of ferromagnetism and unconventional spintriplet superconductivity of Landau-Ginzburg type was derived [11,12] on the basis of general symmetry group arguments. It describes the possible low-order coupling between the superconducting and ferromagnetic order parameters and establishes several important features of the superconducting vortex state in the ferromagnetic phase of unconventional ferromagnetic superconductors $[11,12]$. Both experimental and theoretical problems of ferromagnetism in spin-triplet superconductors seem to be quite different from those in conventional ( $s$-wave) ferromagnetic superconductors $[7,8]$.

In this letter we investigate the conditions for the occurrence of the homogeneous (Meissner-like) phase of coexistence of spin-triplet superconductivity and ferromagnetism in case of a cubic $\left(\mathcal{O}_{h}\right)$ crystal structure. Such a phase of coexistence may occur for a relatively small magnetization and in a zero external magnetic field. Bearing in mind this circumstance and using the model considered in Refs. $[11,12]$ we show that the phase transition to the superconducting state in ferromagnetic superconductors can be either of first or second order and this depends on the model parameters that correspond to the particular substance. Our investigation is based on the mean-field approximation [13] as well as on familiar results about the possible phases in nonmagnetic superconductors with triplet ( $p$-wave) Cooper pairs $[14,15]$. We neglect all anisotropies, usually given by the respective additional Landau invariants and gradient terms [9] in the Ginzburg-Landau free energy of unconventional superconductors. The reason is that the inclusion of crystal anisotropy is related with lengthy formulae and a multivariant analysis which will obscure our main aims and results. Let us emphasize that the present results should be valid in the same or modified form when the crystal anisotropy is properly taken into account. We have to mention also that there is a formal similarity between the phase diagram obtained in our investigation and the phase diagram of certain improper ferroelectrics [16].

\section{MODEL}

We consider the Ginzburg-Landau free energy $[11,12]$ $F=\int d^{3} x f(\psi, \overrightarrow{\mathcal{M}})$, where

$$
\begin{aligned}
f & =\frac{\hbar^{2}}{4 m}\left(D_{j}^{*} \psi\right)\left(D_{j} \psi\right)+a_{s}|\psi|^{2}+\frac{b}{2}|\psi|^{4}+a_{f} \mathcal{M}^{2} \\
& +\frac{\beta}{2} \mathcal{M}^{4}+i \gamma_{0} \mathcal{M} \cdot\left(\psi \times \psi^{*}\right)
\end{aligned}
$$

In Eq. (1), $D_{j}=\left(\nabla-2 i e A_{j} / \hbar c\right)$, and $A_{j}(j=1,2,3)$ are the components of the vector potential $\mathbf{A}$ related with the magnetic induction $\mathbf{B}=\nabla \times \mathbf{A}$. The complex vector $\psi=\left(\psi_{1}, \psi_{2}, \psi_{3}\right) \equiv\left\{\psi_{j}\right\}$ is the superconducting order parameter, corresponding to the spin-triplet Cooper pairing and $\mathcal{M}=\left\{\mathcal{M}_{j}\right\}$ is magnetization. The coupling constant $\gamma_{0}=4 \pi J>0$ is given by the ferromagnetic exchange parameter $(J>0)$. The coefficients 
$a_{s}=\alpha_{s}\left(T-T_{s}\right)$ and $a_{f}=\alpha_{f}\left(T-T_{f}\right)$ are expressed by the positive parameters $\alpha_{s}$ and $\alpha_{f}$ as well as by the superconducting $\left(T_{s}\right)$ and ferromagnetic $\left(T_{f}\right)$ critical temperatures in the decoupled case, when $\mathcal{M} \psi_{i} \psi_{j}$-interaction is ignored; $b>0$ and $\beta>0$, as usual.

We assume that the magnetization $\mathcal{M}$ is uniform, which is a reliable assumption outside quite close vicinity of the magnetic phase transition but keep the spatial ( $\mathrm{x}-$ ) dependence of $\psi$. The reason is that the relevant dependence of $\psi$ on $\mathbf{x}$ is generated by the diamagnetic effects arising from the presence of $\mathcal{M}$ and the external magnetic field $\mathbf{H}[11,12]$ rather than from fluctuations of $\psi$ (this effect is extremely small and can be safely ignored). First term in (1) will be still present even for $\mathbf{H}=0$ because of the diamagnetic effect created by magnetization $\mathcal{M}=\mathbf{B} / 4 \pi>0$. As we shall investigate the conditions for the occurrence of the Meissner phase where $\psi$ is uniform, the spatial dependence of $\psi$ and, hence, the first term in r.h.s. of (1) will be neglected. This approximation should be valid when the lower critical field $H_{c 1}(T)$ is greater than the equilibrium value of the magnetization $\mathcal{M}$ in the phase of coexistence of superconductivity and ferromagnetism.

One may take advantage of the symmetry of model (1) and avoid considering equivalent thermodynamic states that occur as a result of the respective continuous symmetry breaking at the phase transition point but have no effect on the thermodynamics of the system. That is why we shall assume for concreteness of our calculation that the magnetization vector is along the $z$-axis: $\mathcal{M}=(0,0, \mathcal{M})$, where $\mathcal{M} \geq 0$. This concrete choice of the direction of the magnetization vector does not restrict the generality of the present analysis and leads to the same structure of the ordered phases as previously predicted and discussed on the basis of general symmetry group considerations in Ref. [12]. We find convenient to use the following notations: $\varphi_{j}=b^{1 / 4} \psi_{j}, \varphi_{j}=\phi_{j} \exp \left(\theta_{j}\right)$, $M=\beta^{1 / 4} \mathcal{M}, \gamma=\gamma_{0} /\left(b^{2} \beta\right)^{1 / 4}, r=a_{s} / \sqrt{b}, t=a_{f} / \sqrt{\beta}$, $\phi^{2}=\left(\phi_{1}^{2}+\phi_{2}^{2}+\phi_{3}^{2}\right)$, and $\theta=\left(\theta_{2}-\theta_{1}\right)$.

We shall not dwell on the metastable and unstable phases described by model (1) [17] nor on the vortex phase $[11,12]$ corresponding to $|\mathbf{B}|>H_{c 1}$. Rather, we consider the stable homogeneous phases in a zero external magnetic field $(\mathbf{H}=0)$ that are described by uniform order parameters $\mathcal{M}$ and $\psi$. We shall essentially use the condition $T_{f}>T_{s}$

\section{RESULTS AND DISCUSSION}

The model (1) describes three stable homogeneous phases: (1) normal phase $\left(\phi_{j}=M=0\right.$ ) (hereafter referred to as N-phase), (2) ferromagnetic (FM-) phase $\left(\phi_{j}=0, M^{2}=-t>0\right)$, and (3) a phase of the coexistence of superconductivity and ferromagnetism (FSphase $)$, given by $\phi_{3}=0, \theta=2 \pi(k-1 / 4),(k=$
$0, \pm 1, \ldots), \phi^{2}=(-r+\gamma M)>0,6 M^{2}>\left(\gamma^{2}-2 t\right)$, and

$$
\frac{\gamma r}{2}=\left(\frac{\gamma^{2}}{2}-t\right) M-M^{3}
$$

This phase of the coexistence has the same symmetry as pointed in Ref. [12] for cubic crystals and in strong spin-orbit coupling limit. It is not difficult to determine the domains of existence and stability of the phases $\mathrm{N}$, FM, and FS. Note, that here we use the term "condition of stability" to indicate the necessary condition of stability when the respective phase corresponds to a minimum of the free energy, i.e., in both cases of stable and metastable states. When a phase corresponds to a global minimum of free energy (a sufficient condition of stability) it will be called a "stable phase". Thus we easily find the following existence and stability regions: $(t>0$, $r>0)$ for the N-phase, $(t<0, r>\gamma M)$ - for FM. In order to obtain the same domain for FS one should consider Eq. (2) together with the additional existence and stability conditions corresponding to this phase: $\gamma M>r$ and $3 M^{2}>M_{0}^{2}$, where $M_{0}>0$ is defined by the $r(M)=0$; see Eq. (2).

Eq. (2) is shown in Fig. 1 for $\gamma=1.2$ and $t=-0.2$. For any $\gamma>0$ and $t$, the stable FS thermodynamic states are given by $r(M)<r_{m}=r\left(M_{m}\right)$ for $M>M_{m}>0$, where $M_{m}$ corresponds to the maximum of the function $r(M)$. Functions $M_{m}(t)$ and $M_{0}(t)=\left(-t+\gamma^{2} / 2\right)^{1 / 2}=$ $\sqrt{3} M_{m}(t)$ are drawn in Fig. 2 for $\gamma=1.2$. Functions $r_{m}(t)=4 M_{m}^{3}(t) / \gamma$ for $t<\gamma^{2} / 2$ (the line of circles in Fig. 3) and $r_{e}(t)=\gamma|t|^{1 / 2}$ for $t<0$ define the borderlines of stability and existence of FS.

In the region on the left of point B (Fig. 3) with the coordinates $\left(-\gamma^{2} / 4, \gamma^{2} / 2\right)$, FS satisfies the existence condition $\gamma M>r$ only below the dashed line $\left[r<r_{e}\right]$. In the domain confined between the lines of circles and the dashed line on the left of point $B$ the stability condition for FS is satisfied but the existence condition is broken. The inequality $r \geq r_{e}(t)$ is the stability condition of FM for $0 \leq(-t) \leq \gamma^{2} / 4$. For $(-t)>\gamma^{2} / 4$ the FM phase is stable for all $r \geq r_{e}(t)$. The dashed line on the left of point B, i. e. for $(-t)>\gamma^{2} / 4$, is a line of the second order FM-FS phase transition. On this line the equilibrium order parameters are given by $\phi_{j}=0$ and $M_{e q}=\sqrt{|t|}$. Therefore, the phase diagram for $(-t)>\gamma^{2} / 4$ is clarified for any $r$. When $r<0$ the FS phase is stable and is described by the function $r(M)$ for $M>M_{0}$, as shown in Fig. 1.

The part of the $t$-axis given by $r=0$ and $t>\gamma^{2} / 2$ in Fig. 3 is a phase transition line of second order that describes the N-FS transition; see, the dashed line to the right of point $\mathrm{A}$ in Fig. 3 The same transition for $0<t<\gamma^{2} / 2$ is represented by the solid line AC which is the equilibrium transition line of the first order phase transition. This equilibrium transition curve is given by the function

$$
r_{e q}(t)=\frac{1}{4}\left[3 \gamma-\left(\gamma^{2}+16 t\right)^{1 / 2}\right] M_{e q}(t),
$$




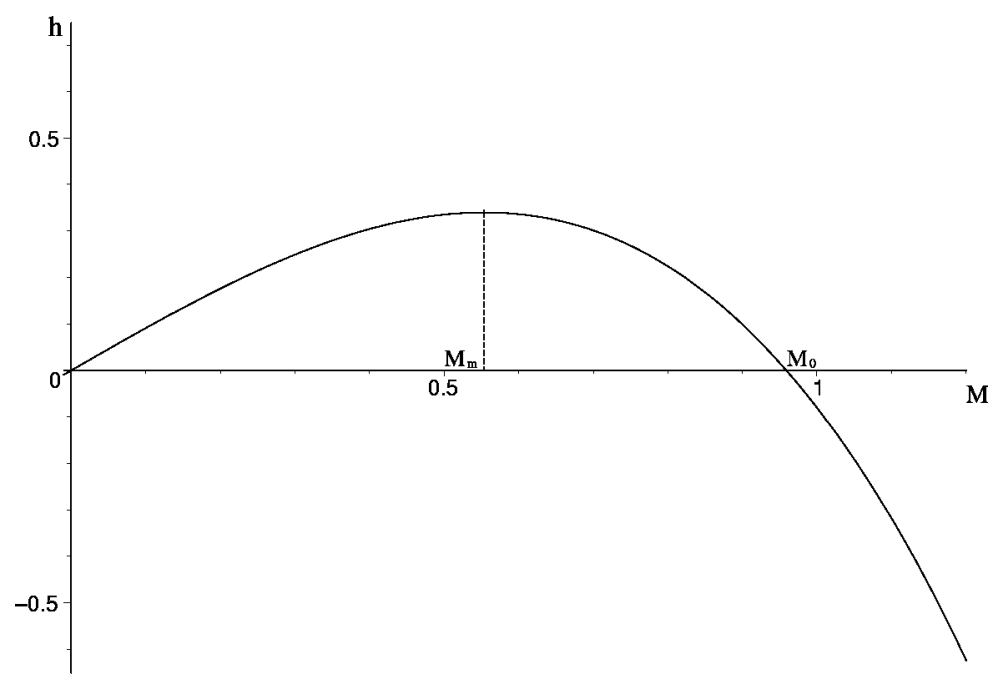

Fig. 1. $h=\gamma r / 2$ as a function of $M$ for $\gamma=1.2$, and $t=-0.2$.

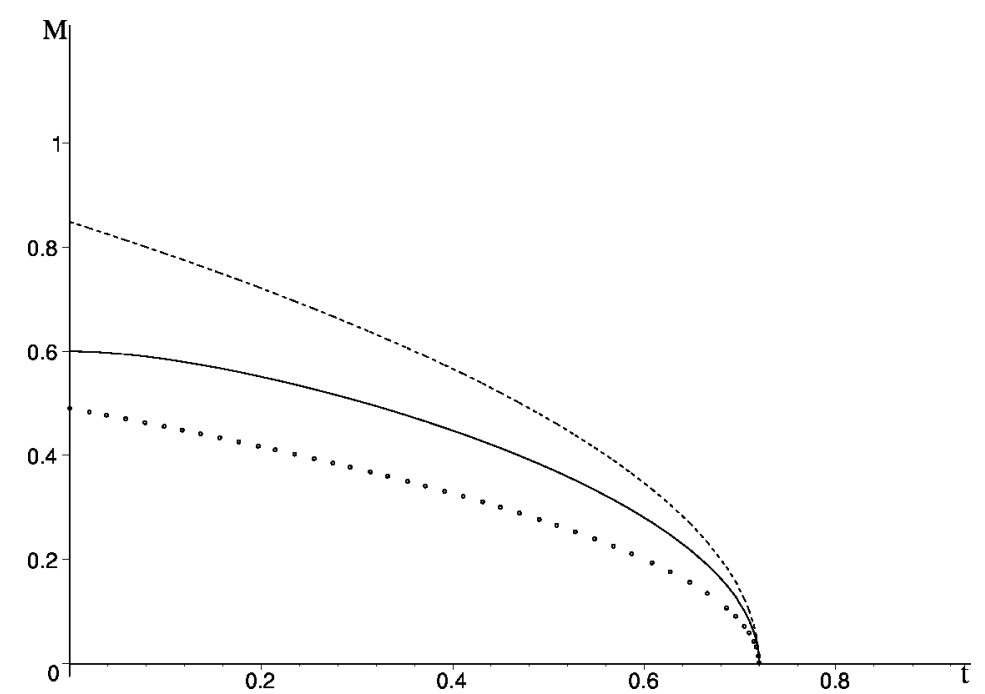

Fig. 2. $M$ versus $t$ for $\gamma=1.2$ : the dashed line represents $M_{0}$, the solid line represents $M_{\mathrm{eq}}$, and the dotted line corresponds to $M_{m}$.

where

$$
M_{\mathrm{eq}}(t)=\frac{1}{2 \sqrt{2}}\left[\gamma^{2}-8 t+\gamma\left(\gamma^{2}+16 t\right)^{1 / 2}\right]^{1 / 2}
$$

is the equilibrium value (jump) of the magnetization. The order of the N-FS transition changes at the tricritical point A.

The domain above the solid line $\mathrm{AC}$ and below the line of circles for $t>0$ is the region of a possible overheating of FS. The domain of overcooling of the N-phase is confined by the solid line $\mathrm{AC}$ and the axes $(t>0, r>0)$. At the triple point $\mathrm{C}$ with coordinates $\left[0, r_{\text {eq }}(0)=\gamma^{2} / 4\right]$ the phases N, FM, and FS coexist. For $t<0$ the straight line

$$
r_{\text {eq }}^{*}(t)=\frac{\gamma^{2}}{4}+|t|, \quad-\gamma^{2} / 4<t<0,
$$

describes the extension of the equilibrium phase transition line of the N-FS first order transition to negative values of $t$, where it describes the FM-FS first order phase transition. For $t<\left(-\gamma^{2} / 4\right)$ the equilibrium phase transition FM-FS is of the second order and is given by the dashed line on the left of point $B$ (the second tricritical point in this phase diagram). Along the first order transition line $r_{\mathrm{eq}}^{*}(t)$, given by (5), the equilibrium 
value of $M$ is $M_{e q}=\gamma / 2$, which implies an equilibrium order parameter jump at the FM-FS transition equal to $(\gamma / 2-\sqrt{|t|})$. On the dashed line of the second order FMFS transition the equilibrium value of $M$ equals that of the FM phase $\left(M_{e q}=\sqrt{|t|}\right)$. At the triple point $\mathrm{C}$ the phases N, FM, and FS coexist. Note, that the dashed line along the $r$-axis above the triple point $\mathrm{C}$ indicates the second order N-FM phase transition.

In conclusion, we have demonstrated that model (1) of ferromagnetic spin-triplet superconductors gives quite a complex phase diagram containing three ordered phases, two types of phase transitions, two tricritical points, and a triple point. Further considerations of the effect of additional terms in free energy (1) such as the terms of the type $\mathcal{M}^{2}|\psi|^{2}$ and/or those describing the Cooper pair and crystal anisotropy $[9,14]$ may give more information about the shape of the phase diagram outlined in the present paper.

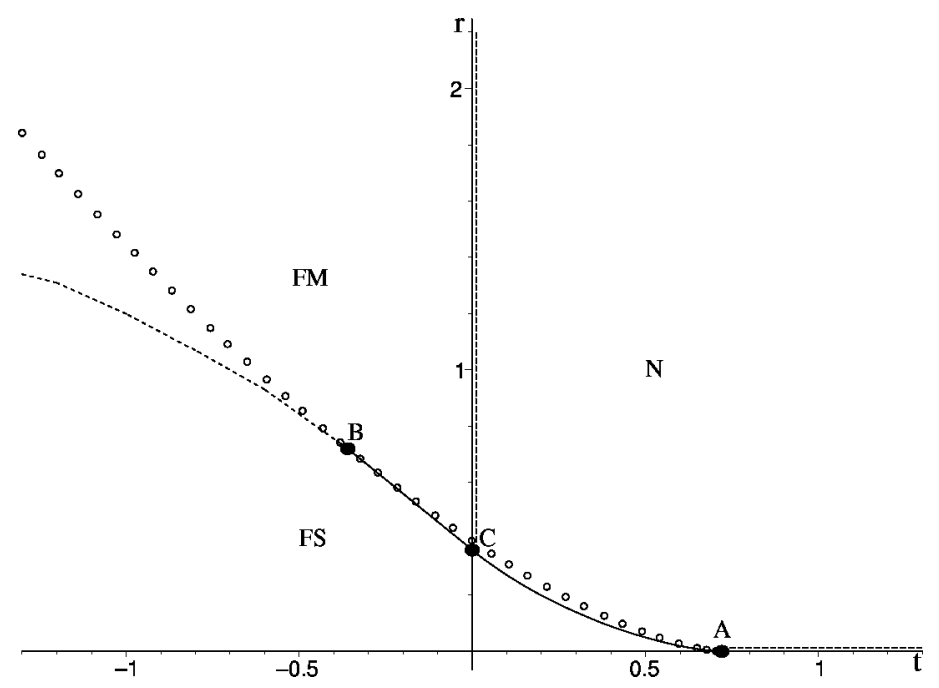

Fig. 3. The phase diagram in the plane $(t, r)$ with two tricritical points (A and B) and a triple point $C ; \gamma=1.2$. The second order transition lines are dashed, first order transition line is solid. The meaning of the notations N, FM and FS is given in the text.

\section{ACKNOWLEDGMENTS}

DIU is thankful for the hospitality of MPI-PKS-Dresden. The financial support through Scenet (Parma) a collaborative project with JINR-Dubna are also acknowledged.

[1] S. S. Saxena, P. Agarwal, K. Ahilan, F. M. Grosche, R. K. W. Haselwimmer, M. J. Steiner, E. Pugh, I. R. Walker, S. R. Julian, P. Monthoux, G. G. Lonzarich, A. Huxley. I. Sheikin, D. Braithwaite, J. Flouquet, Nature (London) 406, 587 (2000).

[2] A. Huxley, I. Sheikin, E. Ressouche, N. Kernavanois, D. Braithwaite, R. Calemczuk, J. Flouquet, Phys. Rev. B 63, 144519 (2001).

[3] N. Tateiwa, T. C. Kobayashi, K. Hanazono, A. Amaya, Y. Haga. R. Settai, Y. Onuki, J. Phys. Condens. Matter 13, L17 (2001).

[4] C. Pfleiderer, M. Uhlatz, S. M. Hayden, R. Vollmer, H. v. Löhneysen, N. R. Berhoeft, G. G. Lonzarich, Nature (London) 412, 58 (2001).

[5] D. Aoki, A. Huxley, E. Ressouche, D. Braithwaite, J. Flouquet, J-P. Brison, E. Lhotel, C. Paulsen, Nature (London) 413, 613 (2001).

[6] K. Shimizu, T. Kimura, S. Furomoto, K. Takeda, K. Kon- tani, Y. Onuki, K. Amaya, Nature (London) 412, 316 (2001).

[7] E. I. Blount, C. M. Varma, Phys. Rev. Lett. 42, 1079 (1979).

[8] T. K. Ng, C. M. Varma, Phys. Rev. Lett. 78, 330 (1997).

[9] V. P. Mineev, K. V. Samokhin, Introduction to Unconventional Superconductivity (Gordon and Breach, Amsterdam, 1999).

[10] S. S. Saxena, P. B. Littlewood, Nature (London) 412 , $290(2001)$.

[11] K. Machida, T. Ohmi, Phys. Rev. Lett. 86, 850 (2001).

[12] M. B. Walker, K. V. Samokhin, Phys. Rev. Lett. 88, 204001 (2002).

[13] D. I. Uzunov, Theory of Critical Phenomena (World Scientific, Singapore, 1993).

[14] E. J. Blagoeva, G. Busiello, L. De Cesare, Y. T. Millev, I. Rabuffo, D. I. Uzunov, Phys. Rev. B 40, 7321 (1990).

[15] D. I. Uzunov, in Adv. Theor. Phys., edited by E. Caian- 
iello (World Scientific, Singapore, 1990), p. 96.

[16] Yu. M. Gufan, V. I. Torgashev, Sov. Phys. Solid State 22, 951 (1980); Fiz. Tverd. Tela (Leningrad) 22, 1629
(1980).

[17] D. V. Shopova, D. I. Uzunov, Phys. Lett. A 313, 139 (2003).

\title{
СПІВІСНУВАННЯ ОДНОРІДНИХ ФАЗ СПІН-ТРИПЛЕТНОЇ НАДПРОВІДНОСТИ I ФЕРОМАГНЕТИЗМУ
}

\author{
Д. ІШопова, Д. Узунов \\ Лабораторія СРСМ, Інститут фізики твердого тіла Г. Наджакова \\ Болгарсвка академія наук, $B G-1784$, Болгарія
}

Співіснування однорідної фази спін-триплетної надпровідности та феромагнетизму досліджено за, допомогою феноменологічної моделі спін-триплетного феромагнетного на,ппровідника. Обговорено застосування отриманих результатів до феромагнетних металів, $\mathrm{UGe}_{2}, \mathrm{ZrZn}_{2}$, URhGe i Fe. 